\title{
Dopamine detection by doped single-walled carbon nanotube biosensors: A theoretical study
}

\author{
Nosrat MADADI MAHANI 1 * (D) \\ 1 Department of Chemistry, Payame Noor University, 19395-4697, Tehran, Iran. \\ * Corresponding Author. E-mail: nmmadady@gmail.com (N.M.M.); Tel. +98-9131989497.
}

Received: 29 December 2018 / Revised: 13 April 2019 / Accepted: 14 April 2019

\begin{abstract}
In this paper, biosensors of the Fe-Nitrogen-doped zigzag $(8,0)$ carbon nanotube and Fe-doped zigzag $(8$, $0)$ carbon nanotube were offered for detection of dopamine molecule. The adsorption property and sensing mechanism of Fe-doped zigzag $(8,0)$ carbon nanotube and Fe-N-SWCNT $(8,0)$ with dopamine were investigated based on density functional theory. The obtained results demonstrated that both Fe-SWCNT and the Fe-N-SWCNT had good adsorption for dopamine, also conductivity also grew when they interacted with it. When dopamine is adsorbed on the surface of the single wall carbon nanotube, a large number of electrons transfer from the Fe-N-doped zigzag $(8,0)$ single carbon nanotube to dopamine, resulting in lessened frontier orbital energy gap and increased electrical conductivity. On the other hand, when dopamine is adsorbed on the surface of the SWCNT, the electrons transfer from dopamine to the FeN-SWCNT, the frontier orbital energy gap rises, while the electrical conductivity declines. Thus, Fe-nitrogen- SWCNT $(8,0)$ is more suitable and sufficient than Fe-SWCNT $(8,0)$ for dopamine detection.
\end{abstract}

KEYWORDS: Doped-single wall carbon nanotube (SWCNT); dopamine; density functional theory (DFT); interaction.

\section{INTRODUCTION}

Given their excellent sensing behaviors including fast response, low priced, good sensitivity, and being environmentally friendly, carbon nanotubes (CNTs) have been engrossed a great deal of attention as a new member of sensors and a novel sensing material applied for gas sensing and detection [1]. As well as, CNTs have a large potential to mediate fast electron-transfer kinetics for a wide range of electroactive species such as hydrogen peroxide, owing to high surface-to-volume ratio [2]. It has been presented that the electronic properties of CNTs can be moderated by vacancy defects electronic field $[3,4]$ filling some species inside the CNTs [5], in addition physical or chemical adsorptions on the side wall of the tubes [5]. On the other hand, non-covalent modification of CNTs has been developed for different uses such as drug delivery, biochemical sensors, gene delivery, and therapeutic applications [6]. An appropriate technique to supply single-walled carbon nanotubes (SWCNTs) to detect gas molecules especially organic chemicals and biological substances is doping with heteroatom substitution. The adsorption sufficiency of SWCNTs can be amender through introducing heteroatom impurities (such as silicon, boron, zinc, titanium and nitrogen) and creating active sites in tube walls [7].

Further, doped CNT sensors can raise their adsorptivity, therewith modifying their sensing property towards gases, due to the strong electron accepting/withdrawing behavior of transition metals [8]. Investigations [9] have suggested that transition metals have a wealthy d-electron and empty orbital, to which the tiny gas molecule can bond strongly when adsorbed on the surface. Newly, carbon nanotube-based sensors have been applied for the detection of biomolecules and other bio-related applications [10]. Dopamine (DA) as one of the most momentous catecholamine neurotransmitters [11], could be involved in many neurological mechanisms, from the extrapyramidal motor tracts of the central nervous system to psychostimulants' addiction [12]. As such, as well as to the relevance of detecting it accurately, also been used as a potent drug for activating receptors in the brain, heart muscle, kidney, and gut [13]. Additionally, it has an important role in detecting emergence of Parkinson's disease, drug abuse, and HIV infection of some cells [14-16]. Necessity of exact and correct detection at physiological levels of dopamine, is the principal factor for the progress of several techniques including electrochemistry [17], chromatography [18], gravimetric [19], and optical

How to cite this article: Madadi Mahani N. Dopamine detection by doped single-walled carbon nanotube biosensors: A theoretical study. J Res Pharm. 2019; 23(5): 785-791. 
spectroscopy [20]. At physiological levels, the dominant presence of ionized DA has been reported from both experimental [21] and theoretical [22] results.

Carbon nanospikes (CNS) on several types of metal wires to develop an electrochemical sensor for dopamine detection have investigated by Alexander et al. [23]. Sansuc et al. have developed ultrasensitive detection of dopamine using a CNT network microfluidic flow electrode [24]. A graphene-modified acupuncture needle for dopamine detection has introduced by Tang et al. [25]. The efficiency of a SWCNT modified gold electrode for dopamine sensing by cyclic voltammetry have been studied by Kurniawan et al. [26]. Also, they investigated interfering effect of biological molecules like ascorbic acid (AA) in determining dopamine and have not observed anyinterference signal from ascorbic acid (AA) during DA etection. Ab initio first principles methods have been used to probe the adsorption mechanism of $\mathrm{NO}_{2}$ gas on $\mathrm{M}$-doped $(\mathrm{Ti}, \mathrm{Cu}$, and Pt) SWCNT $(8,0)$ to determine the most efficient combination for $\mathrm{NO}_{2}$ sensing [27]. A density functional theory (DFT) study has been performed on Rh-doped CNT biosensors for prediagnosis of lung cancer and detection of $\mathrm{CO}_{2}, \mathrm{C}_{6} \mathrm{H}_{6}$ and $\mathrm{C}_{6} \mathrm{H}_{7} \mathrm{~N}$ gases [28]. Also, another DFT study has been performed on a new novel bionanosensor hybrid of tryptophan/Pd doped single walled carbon nanotube [29].

Density functional theory calculations by Yeh et al. has performed for study of adsorption of dopamine on semiconducting $(6,5)$ SWNT, including pure, and B, N co-doped $(6,5)$ SWNTs [30]. Their results have indicated that DA can form multiple molecular interactions with pure $(6,5)$ SWNT. SWCNTs have been modified using Fe and nitrogen to improve their intrinsic characteristics, where this modification has become a research hotspot. The DFT calculation-based method GGA has been conducted to study the interaction of dopamine and nitrogen-Fe doped single carbon nanotube $(8,0)$.

\section{RESULTS AND DISCUSSION}

The Fukui indices enable for identifying the reactive regions as well as the electrophilic and nucleophilic behavior of a molecule along with its chemical reactivity. For a limited system, when a molecule is accepting electrons, it has the Fukui index for nucleophilic attack $\left(f^{+}\right)$, while when the molecule is donating electrons, it has the Fukui index for electrophilic attack (f-). Accordingly, HOMO and LUMO are related to negative and positive Fukui indices, respectively:

$$
\begin{aligned}
& f^{+}(r)=\left(\frac{\partial \rho(r)}{\partial N}\right)_{v(r)}^{+}=\rho(r)_{N+1}-\rho(r)_{N}=\left|\varphi_{\text {LUMO }}(r)\right|^{2}=\rho_{\text {LUMO }}(r) \\
& f^{-}(r)=\left(\frac{\partial \rho(r)}{\partial N}\right)_{v(r)}^{-}=\rho(r)_{N}-\rho(r)_{N-1}=\left|\varphi_{\text {Номо }}(r)\right|^{2}=\rho_{\text {Номо }}(r)
\end{aligned}
$$

The Fukui indices permit forecasting where the most electrophilic and nucleophilic sites of the drug molecule are. Fukui indices calculations as well as DFT-based reactivity descriptor were used in the characterization of the preferred reactive sites, thereby providing a firm explanation for the reactivity of the dopamine molecule.

The Fukui indices for the dopamine molecule in the gas phase are presented in Figure 1. In this figure, the $\mathrm{O}(0.121)$ atom represents the nucleophilic sites of the molecule. This figure shows that the Fukui indices (f-) are scattered throughout almost the entire molecule. The N1 (0.135) and C2 (0.124) atoms have the highest nucleophilic electron density and the highest electrophilic electron density.

The Fukui indices for the dopamine molecule in the solution phase almost similar to Fukui indices in the gas phase. After geometric optimization, the obtained model of Fe-CNT and dopamine molecule was incorporated into the same space to precede the adsorption processes, i.e. initiating the integral optimization of the whole system, with the final relaxed configurations displayed in Figure 2(b). The adsorption energy of dopamine and Fe-SWCNT has been $-3.2141 \mathrm{eV}$, whereas the adsorption energy of dopamine and Fe-NSWCNT $(8,0)$ has been obtained as $-3.6286 \mathrm{eV}$. İn solution phase, the adsorption energy of dopamine and FeSWCNT has been gained $-3.2652 \mathrm{eV}$, whereas the adsorption energy of dopamine and Fe-N-SWCNT $(8,0)$ has been gained as $-3.7362 \mathrm{eV}$.

These data display that the adsorptions are exothermic and spontaneous. The negative values of adsorption energies indicate the strong interaction in these systems; the more negative these values are, the more easily the process would occur. More specifically, such interactions can be conceived as chemisorption due to the large Ead. The energy gap $\left(E_{\mathrm{g}}\right)$ of Fe-CNT/DA system decreased to $0.096 \mathrm{eV}$ from $0.099 \mathrm{eV}$ for pure Fe-CNT system as presented in Table 1, suggesting the growing conductivity for Fe-CNT after dopamine adsorption. 
Table 1. $E_{\mathrm{HOMO}}, E_{\mathrm{LUMO}}$ and $E_{\mathrm{g}}{ }^{*}$ of different systems.

\begin{tabular}{|c|c|c|c|c|c|c|}
\hline \multirow[t]{2}{*}{ System } & \multicolumn{2}{|c|}{$\mathrm{E}_{\text {HOMо }}(\mathrm{eV})$} & \multicolumn{2}{|c|}{$\mathrm{E}_{\text {LUMO}}(\mathrm{eV})$} & \multicolumn{2}{|c|}{$\mathrm{E}_{\mathrm{g}}(\mathrm{eV})^{*}$} \\
\hline & Gas phase & $\begin{array}{c}\text { Solution } \\
\text { phase }\end{array}$ & Gas phase & $\begin{array}{c}\text { Solution } \\
\text { phase }\end{array}$ & Gas phase & $\begin{array}{c}\text { Solution } \\
\text { phase }\end{array}$ \\
\hline Fe-SWCNT & -3.792 & -3.961 & -3.693 & -4.001 & 0.099 & 0.102 \\
\hline N-Fe-SWCNT & -3.654 & -4.089 & -3.561 & -3.956 & 0.093 & 0.088 \\
\hline Fe-SWCNT-DA & -3.608 & -4.047 & -3.512 & -3.995 & 0.096 & 0.091 \\
\hline (N-Fe-SWCNT)-DA & -3.791 & -4.081 & -3.707 & -4.001 & 0.084 & 0.086 \\
\hline
\end{tabular}

Also, in solution phase, energy gap $\left(E_{\mathrm{g}}\right)$ of Fe-CNT/DA system decreased from 0.102 to 0.091 for pure Fe-CNT system.

The density distributions of $\mathrm{HOMO}$ and LUMO changed to $-3.608 \mathrm{eV}$ for $E_{\mathrm{HOMO}}$ and $-3.512 \mathrm{eV}$ for $E_{\mathrm{LUMO}}$. With regard to Table 1 , the energy gap $\left(E_{\mathrm{g}}\right)$ of Fe-N-SWCNT $(8,0) / \mathrm{DA}$ system decreased to $0.084 \mathrm{eV}$ from $0.093 \mathrm{eV}$ for pure Fe-N-SWCNT system, revealing the enhancing conductivity for Fe-N-SWCNT after dopamine adsorption. Also, İn solution phase, energy gap $\left(E_{\mathrm{g}}\right)$ of Fe-CNT/DA system decreased from 0.088 to 0.086 for pure Fe-CNT system.

Also, the density distributions of $\mathrm{HOMO}$ and LUMO changed to $-3.791 \mathrm{eV}$ for $E_{\mathrm{HOMO}}$ and $-3.707 \mathrm{eV}$ for ELUMO.

(a)

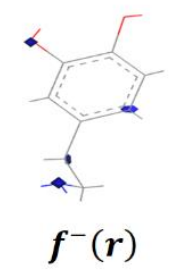

(b)

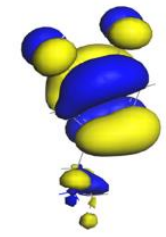

HOMO

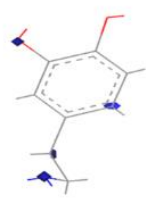

$\boldsymbol{f}^{+}(\boldsymbol{r})$

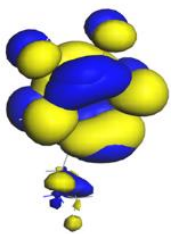

LUMO

Figure 1. Fukui indices, HOMO and LUMO distributions of dopamine.

Note that after adsorption, the density of HOMO and LUMO distribution has transformed particularly those areas near the Fe and $\mathrm{N}$ atoms, with the underlying assumption that strong electronic transfer occurred surrounding the $\mathrm{Fe}$ and $\mathrm{N}$ dopants. The $\mathrm{Fe}$ and $\mathrm{N}$ atoms can provide some activated sites for the dopamine molecule adsorption causing density redistribution of the two orbitals thereby leading to conductivity alteration of Fe-N-SWCNT after the adsorption process. The orbital distributions of each system are shown in Figure 2. Figure 3 demonstrates that the LUMO is almost distributed throughout the molecule 1, while the HOMO is distributed across the molecule.

Also, the HOMO and LUMO move toward the nucleus whereby the orbital energy drops. Fe-SWCNT and Fe-SWCNT are a p-type semiconductor. The transferred electrons increase the number of electron holes and simultaneously raise the number of carriers, enhance the conductivity, and reduce the frontier orbital energy gap. The presence of an iron and nitrogen atoms would create the suitable spaces on the nanotube for the adsorption between dopamine and carbon nanotubes. The results both gas and solution phases, revealed that the adsorption of dopamine on the outer surface of carbon nanotubes was energetically favorable and the reactivity of Fe-N- doped complexes increased compared to pristine carbon nanotubes. 

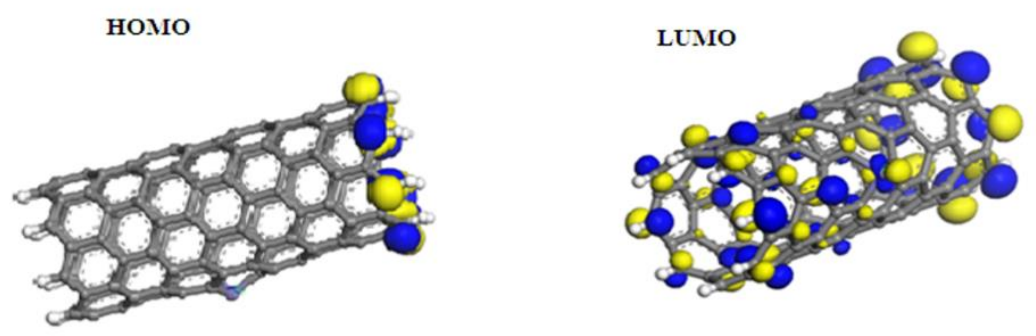

(a)

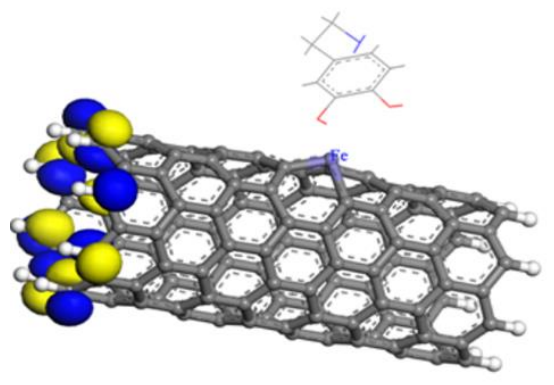

(b)

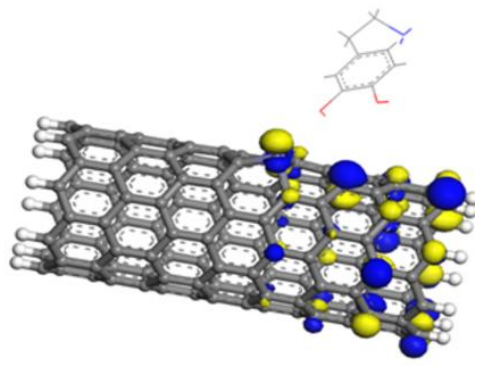

Figure 2. HOMO and LUMO distributions of Fe-CNT (a) and Fe-CNT-DA (b).
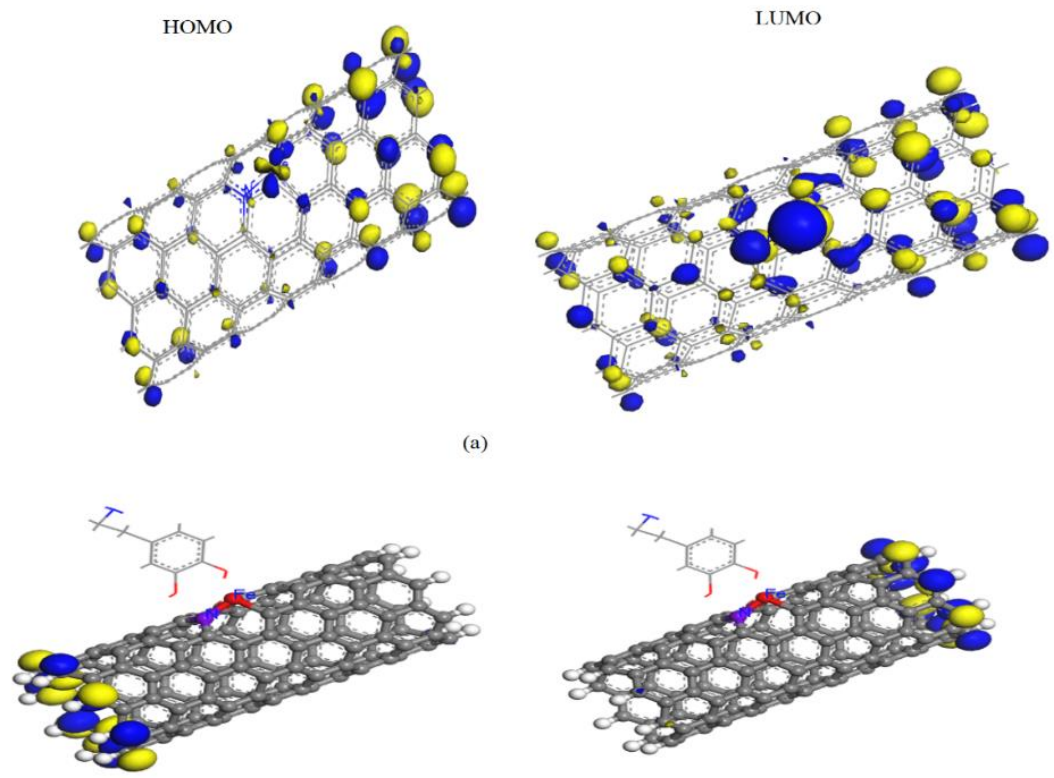

(b)

Figure 3. HOMO and LUMO distributions of Fe-N-SWCNT (a) and Fe-N-SWCNT-DA (b).

\section{CONCLUSION}

This work simulated the adsorption behaviors of Fe-SWCNT and Fe-N-SWCNT $(8,0)$ upon dopamine molecule addition to detect dopamine with DFT approach. Also, the sensitivity of the Fe-SWCNT and Fe-NSWCNT to dopamine was investigated. The interactions between the Fe-SWCNT and Fe-N-SWCNT and dopamine were exothermic; however, the adsorption energy of Fe-N-SWCNT was more satisfactory. Further, the frontier orbital energy gap diminished. The theoretical calculation results inferred that Fe-N-SWCNT can have good sensitivity to the dopamine molecule. Thus, both Fe- SWCNT and Fe-N-SWCNT $(8,0)$ can be suitable options for dopamine molecule detection. These results are helpful in the development of CNT-based sensors and provides a theoretical basis for developing Fe-N-SWCNT-based sensors; the outcomes also 
indicated that the Fe- and N-doped adsorbents had a superior sensing property. The aim of this study was to suggest desirable sensing materials for future application as biosensors in the field of disease diagnosis and detection of drugs.

\section{MATERIALS AND METHODS}

All calculations were performed using $\mathrm{DMol}^{3}$ code [31] which is based on DFT. Consequently, the electronic wave functions were extended in numerical atomic basis sets explained an atomic-centered spherical polar mesh. The electron density in $\mathrm{DMol}^{3}$ was extended in terms of atomic-centered partial densities with multipolarity capable of carrying out both all-electron or pseudo-potential calculations. The double numerical plus d-function all electron basis set was employed for calculations. The DND basis set involves one numerical function for each occupied atomic orbital and a second set of functions for valance atomic orbitals, plus a polarization d-function on all atoms. This basis set is comparable with the Gaussian 6-31G*. The excellent modality of these basis sets minimizes basis set superposition effects and authorized better explanation of molecular polarizabilities. The density function is treated within the generalized gradient approximation with exchange correlation potential demonstrated by Wang and Perdew method (GGA-PW91) [32].

Conductor-like Screening Model (COSMO) as implemented in $\mathrm{DMol}^{3}$ [33] has been chosen for calculation in solution phase, which allow for the treatment of solvation effects. The deviations of this COSMO approximation from the exact solution are small. Firstly, the $(80)$ SWCNT was selected as the pristine model of the SWCNT, based on which Fe-SWCNT and N-Fe-SWCNT were developed. When Fe is doped on the perfect surface of a pristine nanotube and a nitrogen-single nanotube or away from the surface defects, the interaction between the Fe and the nanotube is weak.

Further, this is difficult that constructing a stable structure for adsorption. When Fe is adsorbed on the point defect site of the nanotube surface, the structure becomes stable. Initially, the Fe-SWCNT model was built in this stable structure, with the geometry optimization structure demonstrated in Figure 2(a). The radius of the Fe atom is remarkably greater than that of the C atom; thus, the Fe atom of a Fe-SWCNT is highlighted on the nanotube surface. The bond lengths between the Fe atom and the three neighbor $\mathrm{C}$ atoms are 0.14178 (Fe -C1), $0.14172(\mathrm{Fe}-\mathrm{C} 2)$, and $0.14173 \mathrm{~nm}(\mathrm{Fe}-\mathrm{C} 3)$.

The adsorption energy $\left(\mathrm{E}_{\mathrm{ads}}\right)$ between the dopamine molecule and nanotube can be obtained using the following formula:

$$
E_{\text {ads }}=E_{\text {System }}-E_{\mathrm{Fe}-\mathrm{SWCNT}}-E_{\text {dopamine }}
$$

Where, $E_{\text {ads }}$ represents the total energy of the adsorption structure, $E_{\mathrm{Fe}-\mathrm{SWCNT}} \mathrm{T}$ is the energy of FeSWCNT, and $E_{\text {dopamine }}$ is the energy of one isolated dopamine molecule.

In the same vein, the Fe-Nitrogen SWCNT model was built in this stable structure, with the geometry optimization structure displayed in Figure 3(a). The radii of the Fe atom and nitrogen atom are remarkably greater than that of the $\mathrm{C}$ atom; thus, Fe and $\mathrm{N}$ atoms in Fe-N -SWCNT are highlighted on the nanotube surface.

The adsorption energy ( $\mathrm{E}_{\mathrm{ads}}$ ) between the dopamine molecule and the nanotube can be calculated using the following formula:

$$
E_{\text {ads }}=E_{\text {system }}-E_{\mathrm{Fe}-\mathrm{N}-\mathrm{SWCNT}}-E_{\text {dopamine }}
$$

Where, $E_{\text {ads }}$ represents the total energy of the adsorption structure, $E_{\mathrm{Fe}-\mathrm{N}-\mathrm{SWCNT}}$ shows the energy of Fe-N-SWCNT, and $E_{\text {dopamine }}$ denotes the energy of one isolated dopamine molecule. All values of energies are obtained by optimization of the geometry structure. In addition to energy, we also calculated the electronic properties obtained from the local and net charge transfers as well as the electron density according to the Milliken charge population. 
Acknowledgements: I gratefully thank Payame Noor University for financial support. The authors would like to express their appreciations to department of chemistry this university for providing research facilities.

Author contributions: Concept - N.M.M.; Design - N.M.M.; Supervision - N.M.M.; Materials - N.M.M.; Data Collection and/or Processing - N.M.M.; Analysis and/ or Interpretation - N.M.M.; Literature Search - N.M.M.; Writing - N.M.M.; Critical Reviews - N.M.M.

Conflict of interest statement: The author declared no conflict of interest.

\section{REFERENCES}

[1] Moradi R, Sebt SA, Karimi-Maleh H, Sadeghi R, Karimi F, Bahari A, Arabi H. Synthesis and application of FePt/CNTs nanocomposite as a sensor and novel amide ligand as a mediator for simultaneous determination of glutathione, nicotinamide adenine dinucleotide and tryptophan. Phys Chem Chem Phys. 2013; 15(16): 5888-5897. [CrossRef]

[2] Wang J, Musameh M, Lin Y. Solubilization of carbon nanotubes by nafion toward the preparation of amperometric biosensors. J Am Chem Soc. 2003; 125(9): 2408-2409. [CrossRef]

[3] Collins PG, Bradley K, Ishigami M, Zettl A. Extreme oxygen sensitivity of electronic properties of carbon nanotubes. Science. 2000; 287(5459): 1801-1804. [CrossRef]

[4] Zhang X, Cui H, Gui Y, Tang J. Mechanism and application of carbon nanotube sensors in $\mathrm{SF}_{6}$ decomposed production detection: A review. Nanoscale Res Lett. 2017; 12(1): 1945-1948. [CrossRef]

[5] Zhang X, Cui H, Gui Y, Tang J. Adsorption characteristic of Pd-4 cluster carbon nanotube towards transformer oil dissolved components: A simulation. Appl Surf Sci. 2017; 419: 802-810. [CrossRef]

[6] Asuri P, Bale SS, Pangule RC, Shah DA, Kane RS, Dordick JS. Structure, function, and stability of enzymes covalently attached to single-walled carbon nanotubes. Langmuir. 2007; 23(24): 12318-12321. [CrossRef]

[7] Bennet KE, Tomshine JR, Min HK, Manciu FS, Marsh MP, Paek SB, Settell ML, Nicolai EN, Blaha CD, Kouzani AZ, Chang SY, Lee KH. A Diamond-based electrode for detection of neurochemicals in the human brain. Front Hum Neurosci. 2016; 10: 102-112. [CrossRef]

[8] Zhang $\mathrm{X}$, Dai Z, Chen Q, Tang J. A DFT study of $\mathrm{SO}_{2}$ and $\mathrm{H}_{2} \mathrm{~S}$ gas adsorption on Au-doped single-walled carbon nanotubes. Phys Scr. 2014; 89(6): 065803. [CrossRef]

[9] Sharafeldin IM, Allam NK. DFT insights into the electronic properties and adsorption of $\mathrm{NO}_{2}$ on metal-doped carbon nanotubes for gas sensing applications. New J Chem. 2017; 41(24): 14936-14944. [CrossRef]

[10] Matyszewska D, Napora E, Zelechowska K, Biernat JF, Bilewicz R. Synthesis, characterization, and interactions of single-walled carbon nanotubes modified with doxorubicin with Langmuir-Blodgett biomimetic membranes. J Nanopart Res. 2018; 20(5): 143-159. [CrossRef]

[11] Li H, Wang X, Yu Z. Electrochemical biosensor for sensitively simultaneous determination of dopamine, uric acid, guanine, and adenine based on poly-melamine and nano Ag hybridized film-modified electrode. J Solid State Electrochem. 2014; 18(1): 105-113. [CrossRef]

[12] Ciano PD, Foll BL, Grandy D. Dopamine D4 receptors in psychostimulant addiction. Adv Pharmacol. 2014; 69: 30121. [CrossRef]

[13] Stewart AJ, Hendry J, Dennany L. Whole blood electrochemiluminescent detection of dopamine. Anal Chem. 2015; 87(23): 11847-11853. [CrossRef]

[14] Heidbreder CA, Lacroix L, Atkins AR, Organ AJ, Murray S, West A, Shah AJ. Development and application of a sensitive high performance ion-exchange chromatography method for the simultaneous measurement of dopamine, 5-hydroxytryptamine and norepinephrine in microdialysates from the rat brain. J Neurosci Methods. 2001; 122: 135144. [CrossRef]

[15] Maouche N, Ktari N, Bakas I, Fourati N, Zerrouki C, Seydou M, Maurel F, Chehimi MM. A surface acoustic wave sensor functionalized with a polypyrrole molecularly imprinted polymer for selective dopamine detection. J Mol Recognit. 2015; 28(11): 667-678. [CrossRef]

[16] Qiu C, Bennet KE, Tomshine J R, Hara S, Ciubuc JD, Schmidt U, Durrer W, Malcolm BM, Michael E, Felicia M. Ultrasensitive detection of neurotransmitters by surface enhanced raman spectroscopy for biosensing applications. Biointerface Res Appl Chem. 2017; 7: 1921-1926.

[17] Kim YR, Bong S, Kang Y-J, Yang Y, Mahajan RK, Kim JS, Kim H. Electrochemical detection of dopamine in the presence of ascorbic acid using graphene modified electrodes. Biosens Bioelectron. 2010; 25(10): 2366-2369. [CrossRef] 
[18] Koyun O, Gursu H, Gordo S, Sahin Y. Highly Sensitive Electrochemical determination of dopamine with an overoxidized polypyrrole nanofiber pencil graphite electrode. Int J Electrochem Sci. 2017; 12: 6428-6444. [CrossRef]

[19] Mazouz Z, Rahali S, Fourati N, Zerrouki C, Aloui N, Seydou M, Yaakoubi N, Chehimi MM, Othmane A, Kalfat R. Highly selective polypyrrole MIP-based gravimetric and electrochemical sensors for picomolar detection of glyphosate. Sensors. 2017; 17(11): 2586-2597. [CrossRef]

[20] Ciubuc JD, Bennet KE, Qiu C, Alonzo M, Durrer WG, Manciu FS. Raman Computational and experimental studies of dopamine detection. Biosensors. 2017; 7(4): 43-51. [CrossRef]

[21] Jackowska K, Krysinski P. New trends in the electrochemical sensing of dopamine. Anal Bioanal Chem. 2013; 405: 3753-3771. [CrossRef]

[22] Lu JF, Yu ZY. Theoretical determinations of ionization potentials of dopamine. Russ J Phys Chem A. 2013; 87(4): 628633. [CrossRef]

[23] Zestos AG, Yang C, Jacobs CB, Hensley D, Jill Venton B. Carbon nanospikes grown on metal wires asmicroelectrode sensors for dopamine. Analyst 2015, 140:7283-7292. [CrossRef]

[24] Sansuk S, Bitziou E, Joseph MB, Covington JA, Boutelle MG, Unwin PR, Macpherson JV. Ultrasensitive detection of dopamine using a carbon nanotube network microfluidic flow electrode. Anal Chem. 2013; 85 (1): 163-169. [CrossRef]

[25] Tang L, Du D, Yang F, Liang Z, Ning Y, Wang, H, Zhang, GJ. Preparation of graphene-modified acupuncture needle and its application in detecting neurotransmitters. Sci Rep. 2015; 5: 11627-11636. [CrossRef]

[26] Kurniawan F, Kiswiyah NSA, Madurani KA, Tominaga M. Single-walled carbon nanotubes-modified gold electrode for dopamine detection. ECS J Solid State Sci Technol. 2017; 6; M3109-M3112.

[27] Sonawane MR, Nagare BJ, Habale D, Shivade RK. Comparative study of adsorption of $\mathrm{O}_{2}, \mathrm{CO}_{2}, \mathrm{NO}_{2}$ and $\mathrm{SO}_{2}$ on pristine and Si-doped carbon nanotubes. Adv Mat Res. 2013; 678: 179-184. [CrossRef]

[28] Wan Q, Xua Y, Chen X, Xiao H. Exhaled gas detection by a novel Rh-doped CNT biosensor for prediagnosis of lung cancer: A DFT study. Mol Phys. 2018; 116(17): 2205-2212. [CrossRef]

[29] Yeh CH, Hsiao YJ, Jiang JC. Dopamine sensing by boron and nitrogen Co-doped single-walled carbon nanotubes: A first-principles study. App Sur Sci. 2019; 473: 59-64. [CrossRef]

[30] Yoosefian M, Etminan N. Density functional theory (DFT) study of a new novel bionanosensor hybrid; tryptophan/Pd doped single walled carbon nanotube. Physica E. 2016; 81: 116-121.

[31] Delley B. From molecules to solids with the DMol3 approach. J Chem Phys. 2000; 113: 7756-7764. [CrossRef]

[32] Perdew JP, Wang Y. Accurate and simple analytic representation of the electron-gas correlation energy. Phys Rev B. 1992; 45: 13244-13249. [CrossRef]

[33] Delley B. An all-electron numerical method for solving the local density functional for polyatomic molecules. J Chem Phys. 1990; 92(1): 508-517. [CrossRef] 